\title{
State determination of catalytic converters based on an ultra-wideband communication system
}

\author{
I. Motroniuk, R. Stöber, and G. Fischerauer \\ Bayreuth Engine Research Center (BERC), Universität Bayreuth, Bayreuth, Germany \\ Correspondence to: I. Motroniuk (mrt@uni-bayreuth.de)
}

Received: 8 May 2015 - Revised: 16 July 2015 - Accepted: 20 July 2015 - Published: 10 August 2015

\begin{abstract}
A novel microwave-based approach for monitoring the state of aftertreatment systems such as diesel particulate filters (DPFs), three-way catalytic converters (TWCs), and selective catalytic reduction (SCR) catalysts is proposed. The volume inside the metallic housing of the DPF, TWC, or SCR is considered as a wireless communication channel between two terminals of a communication system. It is shown that, depending on the transmission channel characteristics, the properties of the catalyst, such as the catalyst state, can be inferred. This is done by means of an ultra-wideband (UWB) measurement and the subsequent evaluation and processing of the waveform in the time and frequency domains.
\end{abstract}

\section{Introduction}

Modern regulations for exhaust gas components like Euro 5 and Euro 6 require new methods both for the emissions control and for the monitoring and operation of the emission control systems in the diesel and gasoline engines.

During diesel engine operation, soot is captured in the pores of the DPFs (diesel particulate filters) or the SCR (selective catalytic reduction) catalysts. The ceramic filters must be periodically regenerated to avoid blocking. Hence, knowledge about the soot load is useful for the engine control in a number of ways (Feulner et al., 2013; Rose and Boger, 2009; Ochs et al., 2010; Sappok et al., 2010).

For gasoline engines, a TWC (three-way catalytic converter) is used to keep the polluting emissions low. The TWC stores and releases oxygen depending on the operating conditions. As in the DPF case, it would be of great advantage from the control engineering point of view to know the oxygen loading. However, it is the state of the art that the loading state cannot be measured directly but is derived indirectly from the oxygen concentration in the exhaust gas stream before and after the catalytic converter. The oxygen-measuring elements (so-called lambda probes) are implemented for the loading state estimation (Twigg, 2007).

There are a few known approaches for the direct measurement of the desired internal parameters (Zimmermann, 2007; Reiß et al., 2009). These and other similar ones also based on physical sensors can deliver only local information about the catalyst. However, one can learn nothing about the global state, such as the overall loading degree. Additionally, these approaches suffer from the complexities involving the mounting of the sensors in the catalyst and the communication with them.

Since the catalytic converter is typically located in a metallic housing, it and its housing together act as a filled cavity resonator. The resonances are influenced by the changes of material parameters in the catalyst so that the catalyst state can be determined from the appropriate signal characteristics of the resonances. The feasibility of this approach is well documented in the literature (Fischerauer et al., 2008, 2010b; Eichelbaum et al., 2012; Moos et al., 2013). Typically, to observe the cavity resonances, one or two simple thin probe feeds (short stubs) are connected to an automatic vector network analyzer (VNA) via coaxial lines. The changes in the resonant cavity are observed via the scattering matrix parameters $S_{i j}$ measured by the VNA in the laboratory conditions.

It has also been shown that the microwave-based direct observation of TWCs enables superior engine control strategies compared to the indirect observation by way of lambda probes. This superiority consists, for instance, in lower emission levels (Schödel et al., 2014a, b).

The described laboratory equipment cannot be used in the field, i.e., inside a vehicle or in the manufacturing plant, ow- 
ing to space and cost reasons. For the practical application, it should be replaced by smaller and cheaper solutions.

For solving this miniaturizing task, we have described a novel approach towards the monitoring of exhaust gas aftertreatment systems such as DPFs, SCRs, and TWCs (Motroniuk et al., 2014). It was proposed to consider the interior of the catalyst metal housing as a wireless communication channel. The effect of the catalyst state on such communication parameters as the bit error rate (BER), the packet error rate (PER), the ratio of the energy per bit to the noise power spectral density $E_{\mathrm{b}} / N_{0}$ and the data receive rate (Rx data rate) was demonstrated by way of an example. The described approach involves a small-size and cost-effective modular architecture and has the potential for field application, unlike approaches based on laboratory equipment such as automatic vector network analyzers.

In a recent conference contribution, we proposed a modification of our novel approach. Instead of measuring certain communication channel parameters (such as BER, PER, $E_{\mathrm{b}} / N_{0}$ levels, and Rx data rate), an ultra-wideband (UWB) pulse is to be transmitted through the catalyst and the changes suffered by it on its way through the catalyst are to be evaluated by a receiving device (Motroniuk et al., 2015). In other words, the propagation medium characteristics and the catalyst state are to be estimated from the UWB waveform changes.

In this contribution, we consider an extension of the UWB approach for the catalyst state determination. It is based on the subsequent evaluation and post-processing of the UWB waveforms in the time and frequency domains.

The rest of the paper is organized as follows. In Sect. 2, a novel wireless approach is presented, followed by a description of a hardware implementation and of an experimental verification with DPF in the time and frequency domains in Sects. 3 and 4, respectively. The conclusions are presented in Sect. 5.

\section{Novel wireless approach}

It is proposed to consider the interior of the catalyst housing as a communication channel between two terminals of a UWB wireless communication system. The most important parameters during the data transmission are the characteristics of the communication channel (in our case the catalyst). From the wireless channel characteristics, the properties of the catalytic converter such as the catalyst oxygen concentration or soot loading state can be determined. The latter assertion about the measurability of the soot loading state has been demonstrated in Motroniuk et al. (2014) and will be further corroborated in the remainder of this contribution. The former assertion about the measurability of the oxygen concentration follows from the fact that the electrical properties of catalysts depend heavily on their oxidization state; hence, this state can be inferred from the transmission or re-

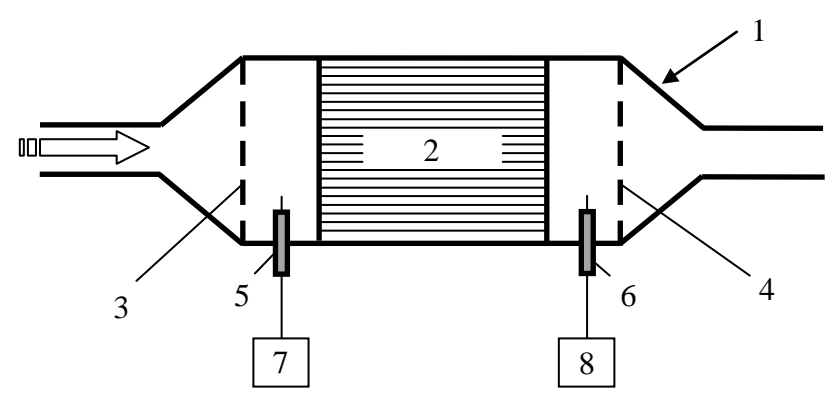

Figure 1. Basic structure of the proposed measurement system.

flection details of electromagnetic microwaves (Fischerauer et al., 2008; Eichelbaum et al., 2012).

The basic structure of the proposed measurement system is shown in Fig. 1. The system consists of a conductive (usually metallic) housing of the catalyst (1) and the catalyst itself (2). Two gas-permeable conductive grids $(3,4)$ which limit the resonating cavity are of advantage but not absolutely necessary. The grids are effectively short-circuiting the electric field, thus precisely defining the resonating cavity. Two thin-probe feeds (short stubs 5, 6) serve to connect the cavity with the system environment, i.e., with communication end devices $(7,8)$, by coaxial cables, which are used for signal transmission and reception. The short stubs play the same role for the communication system as the antennas in the common message transmission and the catalyst in its housing plays the same role as the wireless communication channel for the ordinary message transmission.

The signal transmitted through the wireless channel inside the catalyst suffers from such effects as attenuation, fading, multipath propagation, scattering, etc. Note, however, that the sender and receiver in a common wireless transmission link are located in the far field of each other whereas, in the present context, they are located in the near field. This, together with the resonance phenomena in the catalyst-filled cavity, introduces new propagation-path effects compared to wireless transmission links. The characteristics of the received information transferred from the transmitter to the receiver depend on the propagation medium. The effect on the signal is similar to the effect of buildings, trees, autos, rain, humidity, etc. in common wireless communication links: the channel characteristics influence the signal received, either in the time or frequency domain. Here, the closed-space propagation through the soot-loaded or otherwise chemically altered catalyst medium affects the received signal waveform.

In this paper, we specifically focus on a UWB communication system. Rather than measuring individual channel parameters, such as BER, PER, $E_{\mathrm{b}} / N_{0}$ levels, or the Rx data rate (Motroniuk et al., 2014), or than measuring the $\mathrm{S}$-parameters of a microwave two-port (Fischerauer et al., 2008), it is the integral UWB pulse waveform transmitted through the device under test (catalyst) which is considered. It is postulated that physical changes of the catalyst material 


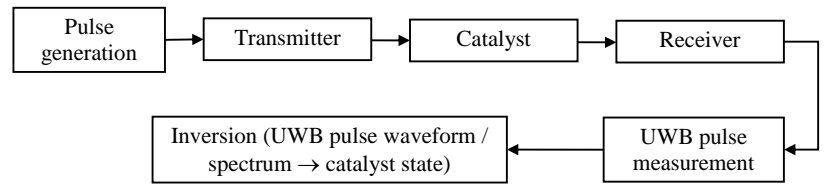

Figure 2. Functional diagram of the proposed measurement system.

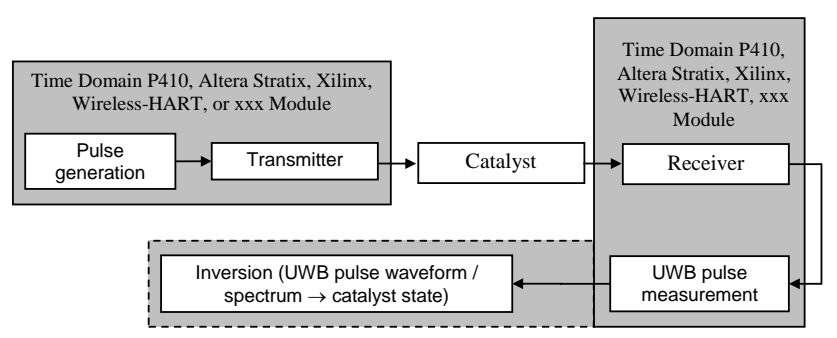

Figure 3. Exemplary hardware implementation of the proposed measurement system.

caused by the absorption or desorption of soot or chemical species are mirrored in the waveform received, or its spectral representation.

The goal, of course, is to estimate the internal state of a catalyst by this approach in a manner substantially simpler than possible with the system architectures demonstrated so far. This is achieved because the measurement can be performed by the existing hardware (wireless communication modules) and software (signal processing techniques for the wireless communication systems).

The functional diagram of such a system is shown in Fig. 2. A pulse generator generates the UWB waveform, which is then transmitted through the device under test (catalyst) and distorted in this process. On the receiver side, the UWB pulse waveform is measured to indirectly estimate the catalyst state.

\section{Hardware implementation}

Figure 3 depicts some possible ways to implement the architecture proposed in actual hardware. The UWB pulse generation and the transmission are accomplished by a communication module (we have used a Time Domain PulsON P410 module), and the receiver is implemented by another communication module (also PulsON P410). After receiving the pulse modulated by the catalyst properties, it is a matter of experience (calibration, simulation results, etc.) to infer the catalyst state from the waveform distortion.

Devices such as Altera Stratix, Xilinx Virtex, Xilinx Spartan, ZigBee, WirelessHART, Nanonet, PulsON 410, or any other including (but not limited to) field-programmable gatearray (FPGA)-based integrated circuits and devices could be used for building an appropriate communication system for the intended application purpose. The catalyst state can be
Table 1. Soot load of the measured DPFs (Fischerauer et al., 2010a).

\begin{tabular}{lrr}
\hline $\begin{array}{l}\text { DPF } \\
\text { number }\end{array}$ & $\begin{array}{r}\text { Soot load } \\
(\mathrm{g})\end{array}$ & $\begin{array}{r}\text { Soot load per DPF } \\
\text { volume }\left(\mathrm{g} \mathrm{L}^{-1}\right)\end{array}$ \\
\hline 1 & 0 & 0 \\
2 & 4.6 & 2.0 \\
3 & 13.6 & 5.8 \\
\hline
\end{tabular}

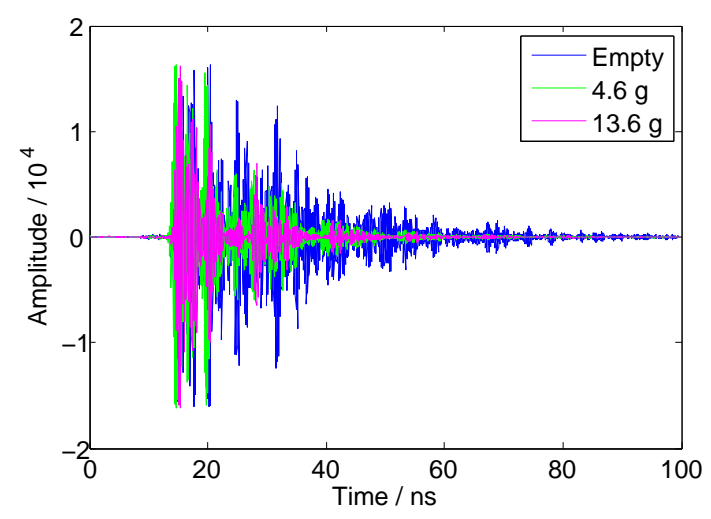

Figure 4. UWB waveforms for three DPFs loaded with different amounts of soot.

estimated by a post-processing of the received UWB waveform with the numerical computing environments and programming languages (such as MATLAB and C).

\section{Experimental verification with DPF}

\subsection{Validation in the time domain}

The validity of the approach was tested with DPFs loaded with different amounts of soot. The aim of the tests was to compare the effect of the soot load on the communication channel and, as a result, on the received UWB waveforms in order to prove the validity of the, as we believe, novel approach towards the monitoring and estimation of catalyst states.

One of the DPFs was clean (not used, with $0 \mathrm{~g}$ of soot), another one was loaded with $4.6 \mathrm{~g}$ of soot, and the third one was loaded with $13.6 \mathrm{~g}$ of soot. The filters used and their soot loading are listed in Table 1. For details of the catalyst geometry, the reader is referred to Fischerauer et al. (2010a).

The UWB pulse waveforms recorded during the communication between two commercial PulsON P410 modules via the interior of the DPF-loaded metal housing look substantially different for the various DPFs (Fig. 4). In particular, multiple-transit signals inside the catalyst housing (occurring after $18 \mathrm{~ns}$ in Fig. 4) are more attenuated the higher the soot load is. This is explained by the fact that the conducting soot introduces losses into the system. Thus, a wave packet is attenuated each time it traverses the soot-loaded DPF, and 


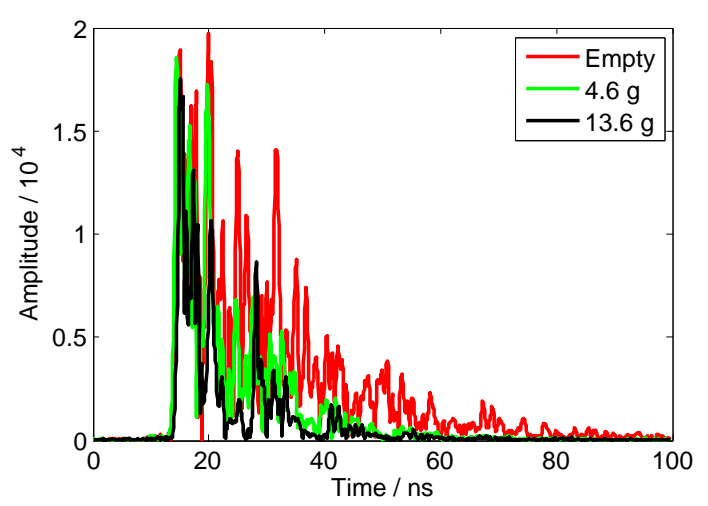

Figure 5. Envelopes of the time-domain responses from Fig. 4.

this attenuates multiple-transit signals more than the singletransit (direct input-to-output) signal. In Fig. 4 the vertical scale represents A-D (analog-to-digital) counts proportional to volts as output by the analog-to-digital converter (ADC) in the UWB chip.

The envelopes $e(t)$ of the time-domain responses shown in Fig. 4 have been computed as the magnitude of the analytic signal constructed from the original time response $u(t)$ by adding to it its Hilbert transform (Marko, 1982):

$e(t)=|u(t)+j \hat{u}(t)|$ with $\hat{u}(t)=\frac{1}{\pi} \int_{-\infty}^{\infty} \frac{u(\tau)}{t-\tau} \mathrm{d} \tau$.

Of course, all signals are discrete in time so that Eq. (1) had to be evaluated in the form

$$
\begin{gathered}
e(k \Delta t)=|u(k \Delta t)+j \hat{u}(k \Delta t)| \\
\text { with } \hat{u}(k \Delta t) \approx \frac{1}{\pi} \sum_{\substack{\ell=\ell_{\min }, \ell \neq k}}^{\ell_{\max }} \frac{u(\ell \Delta t)}{k-\ell} .
\end{gathered}
$$

The result is shown in Fig. 5. One clearly observes a substantial difference in the signal envelopes depending on the soot content in the DPF. In particular, this can be seen starting from the second peek at around $16 \mathrm{~ns}$. The soot loading state is not observable via the first peak because of the technical characteristics of the equipment (at the beginning of the received UWB pulse, the Tx pulse coupling through the Tx switch is showing through).

To quantify the differences, we calculated the convolution of the envelope functions for different DPF states, as always defined by

$$
\begin{aligned}
& c_{i j}(t)=\int_{-\infty}^{+\infty} e_{i}(\tau) e_{j}(t-\tau) \mathrm{d} \tau \quad \text { or } \\
& c_{i j}(k \Delta t) \approx \sum_{\ell=\ell_{\min }}^{\ell_{\max }} e_{i}(\ell \Delta t) e_{j}((k-\ell) \Delta t) \Delta t
\end{aligned}
$$

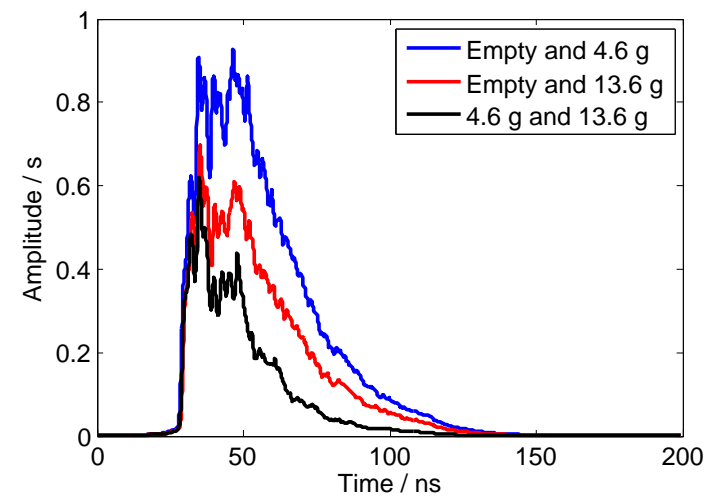

Figure 6. Convolution of the envelopes of the time-domain responses (Fig. 5).

(Fig. 6; when comparing the $y$ axis scaling of this and other figures to the scalings in Motroniuk et al. (2015), note that discrete convolution, correlation, and Fourier-transform formulas were used in the reference, whereas we have used the continuous-signal formulas in the current contribution. For example, the continuous-signal convolution approximately computed by Eq. (2) and the discrete convolution differ by a factor of $\Delta t$ - the sampling interval - and this results in different $y$ axis scalings.) The effect of the soot load on the curves is clearly seen. Multiple-transit signals are more attenuated with increasing soot load. The amplitude of the convolved signals at $50 \mathrm{~ns}$ is around $13 \times 10^{9}$ (A$\mathrm{D}$ counts $) \times 61 \mathrm{ps}(\Delta t) \approx 0.79 \mathrm{~s}$ for the empty/4.6 $\mathrm{g}$ case but only $8.9 \times 10^{9}(\mathrm{~A}-\mathrm{D}$ counts $) \times 61 \mathrm{ps} \approx 0.54 \mathrm{~s}$ for the empty/13.6 g case.

The difference is observable by the width of the convolution curves as well. Measured at an amplitude of $3.28 \times 10^{9}$ (A-D counts) $\times 61 \mathrm{ps}(\Delta t)$, this width amounts to 52,42 , and $27 \mathrm{~ns}$, respectively, for the three cases.

We also calculated the cross-correlation of the envelope functions, given by

$$
\begin{aligned}
& \Phi_{i j}(t)=\int_{-\infty}^{+\infty} e_{i}(\tau) e_{j}(t+\tau) \mathrm{d} \tau \quad \text { or } \\
& \Phi_{i j}(k \Delta t) \approx \sum_{\ell=\ell_{\min }}^{\ell_{\max }} e_{i}(\ell \Delta t) e_{j}((k+\ell) \Delta t) \Delta t .
\end{aligned}
$$

For $i=j$, this turns into the auto-correlation $\Phi_{i i}(t)$ of the envelope function (Fig. 7). In particular, $\Phi_{i i}(0)$ is proportional to the signal power. Figure 7 clearly reveals that the signal power and the other values of the auto-correlation function decrease with the soot load. This is to be expected as more soot means higher signal attenuation, which in turn means smaller signal amplitudes at the receiver. In numbers, the maximum of the auto-correlation function is $3.2 \times 10^{10} \times 61 \mathrm{ps}(\Delta t) \approx 1.96 \mathrm{~s}, 1.8 \times 10^{10} \times 61 \mathrm{ps} \approx 1.11 \mathrm{~s}$, 


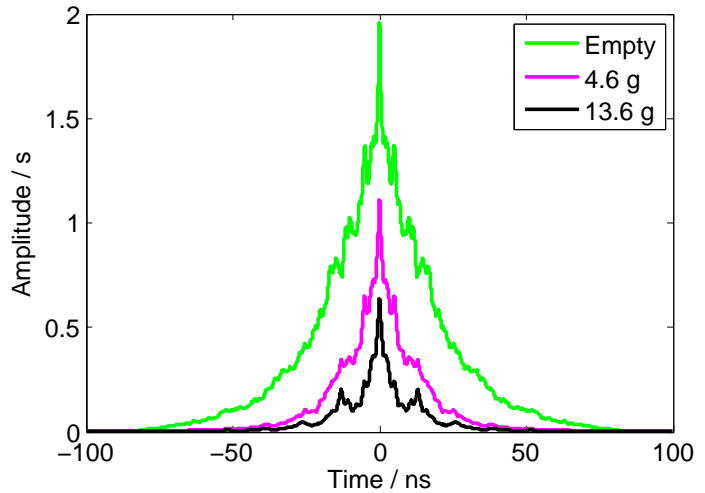

Figure 7. Auto-correlation functions of the envelopes shown in Fig. 5.

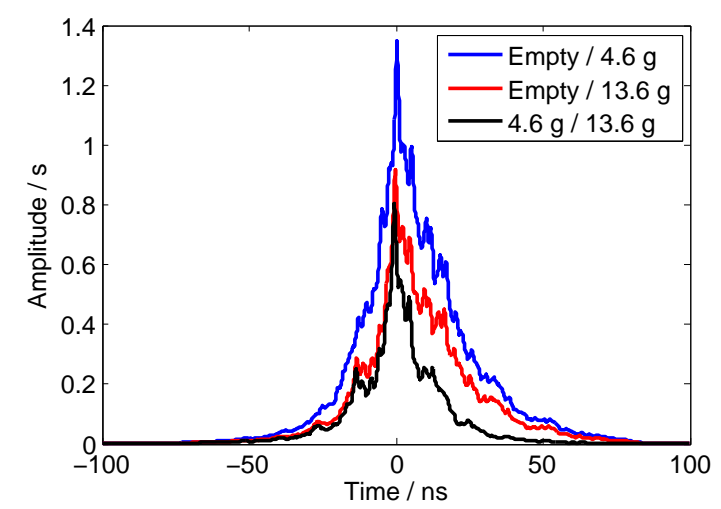

Figure 8. Cross-correlation functions of the envelopes shown in Fig. 5.

and $1 \times 10^{10} \times 61 \mathrm{ps} \approx 0.64 \mathrm{~s}$ for the empty DPF, the DPF loaded with $4.6 \mathrm{~g}$ soot, and the DPF loaded with $13.6 \mathrm{~g}$, respectively. Again, the differences are also observable via the signal width.

Finally, Fig. 8 shows the cross-correlation functions. The amplitudes and mean values of the peaks are among the parameters suitable for a DPF state estimation algorithm. The maximum amplitude was $2.2 \times 10^{10} \times 61 \mathrm{ps}(\Delta t) \approx 1.35 \mathrm{~s}, 1.5 \times 10^{10} \times 61 \mathrm{ps} \approx 0.92 \mathrm{~s}$, and $1.3 \times 10^{10} \times 61 \mathrm{ps} \approx 0.8 \mathrm{~s}$ for the cross-correlations of the three envelope signals from Fig. 5. The mean values were $2.8 \times 10^{9} \times 61 \mathrm{ps} \quad(\Delta t) \approx 0.17 \mathrm{~s}, 1.8 \times 10^{9} \times 61 \mathrm{ps} \approx 0.11 \mathrm{~s}$, and $1.1 \times 10^{9} \times 61 \mathrm{ps} \approx 0.07 \mathrm{~s}$ for the three cases, respectively.

Owing to the rapid amplitude variation of the envelope curves in Fig. 5, it makes sense to first smooth them before further processing (Fig. 9). The amplitude of the smoothed envelopes depends on the soot load - with increasing soot load, the amplitude measured at the receiver becomes lower.

One possible way of processing is to correlate certain features of these smoothed curves with the DPF soot load. This is demonstrated in Fig. 10 for the center of gravity of the ar-

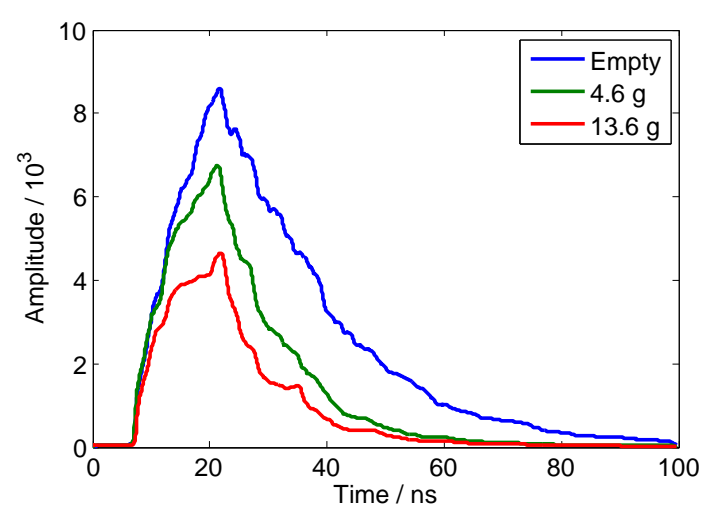

Figure 9. Smoothed envelopes of the time-domain responses from Fig. 4.

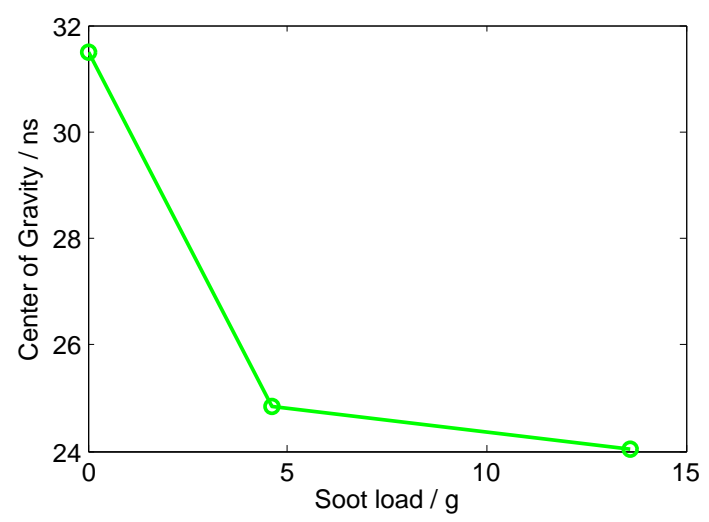

Figure 10. Time coordinate of the center of gravity of the area bounded by the smoothed envelopes and the time axis in Fig. 9.

eas bounded by the curves in Fig. 9 and the time axis. It is obvious that this characteristic curve may be used to estimate the soot load from the measured time-domain response of the UWB system. It is also obvious that the sensitivity of this measurement system decreases with increasing soot load.

Along the same line of reasoning, we have computed the characteristic curves linking the maximum and the average values of the smoothed envelopes with the DPF soot load (Fig. 11).

While the overall behavior of the characteristic curves in Figs. 10 and 11 is quite similar, there are differences in the details. It appears as if small soot loads can be better resolved (measured with a higher sensitivity) by way of the centerof-gravity feature (Fig. 10) than by the other features. This would be quite interesting, but, in our opinion, it is too early to draw such conclusions as the number of measurements we have performed so far is much too small to allow for any substantial insight into the statistics of the measurement approach.

Besides, it is also important to note that the curves will look different with different transmitting power because of 


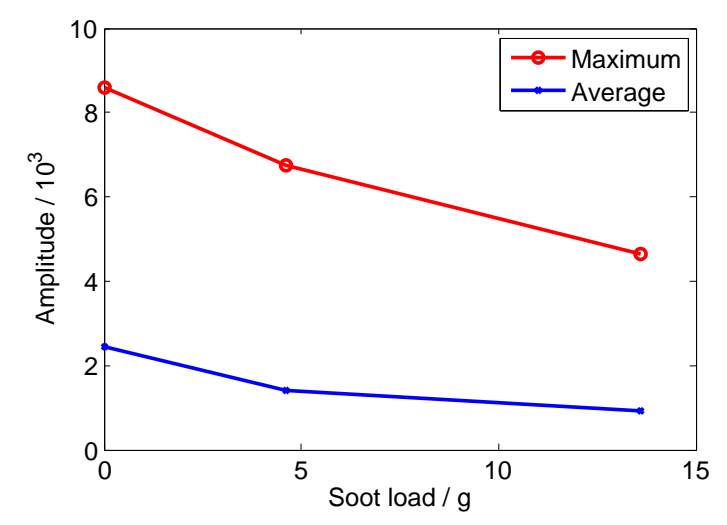

Figure 11. Maximum and average values of the smoothed envelopes (Fig. 9).

receiver sensitivity. This, too, will have to be investigated in more detail.

\subsection{Validation in the frequency domain}

According to the results presented in Sect. 4.1, the state of automotive aftertreatment systems can possibly be inferred from the time-domain characteristics of UWB signals. However, in some cases, it may be easier to characterize and evaluate signals in the frequency domain. This will be considered in the present section.

UWB spectra for the three waveforms from Fig. 4 are shown in Fig. 12 (the spectral range from 3.1 to $5.3 \mathrm{GHz}$ corresponds to the product specifications of the communication units used). One clearly observes substantial differences in the spectral amplitudes between the soot loading degrees, especially at the resonance peaks of 3.18, 3.4, 3.57, 4.0, 4.16, $4.4 \mathrm{GHz}$, etc. For example, the magnitude of the UWB spectrum at $3.4 \mathrm{GHz}$ amounts to $7.6 \times 10^{5}$ (A$\mathrm{D}$ counts $) \times 61 \mathrm{ps}(\Delta t) \approx 4.6 \times 10^{-5} \mathrm{~s}$ for the empty DPF, to $5.9 \times 10^{5} \times 61 \mathrm{ps} \approx 3.6 \times 10^{-5} \mathrm{~s}$ for the $4.6 \mathrm{~g}$ load, and to $4.3 \times 10^{5} \times 61 \mathrm{ps} \approx 2.6 \times 10^{-5}$ s for the $13.6 \mathrm{~g}$ load. Obviously, the signals are more attenuated the higher the soot load is.

The convolution of the UWB magnitude spectra from Fig. 12 with each other is calculated with

$$
\begin{aligned}
& C_{i j}(f)=\int_{-\infty}^{+\infty} U_{i}(\varphi) U_{j}(f-\varphi) \mathrm{d} \varphi \text { or } \\
& C_{i j}(k \Delta f) \approx \sum_{\ell=\ell_{\min }}^{\ell_{\max }} U_{i}(\ell \Delta f) U_{j}((k-\ell) \Delta f) \Delta f
\end{aligned}
$$

and plotted in Fig. 13. The maximum magnitude of the convolved signals in the frequency domain is around $0.29 \mathrm{~s}$ for the empty $/ 4.6 \mathrm{~g}$ case, $0.21 \mathrm{~s}$ for the empty/13.6 g case, and only $0.17 \mathrm{~s}$ for the $4.6 \mathrm{~g} / 13.6 \mathrm{~g}$ case.

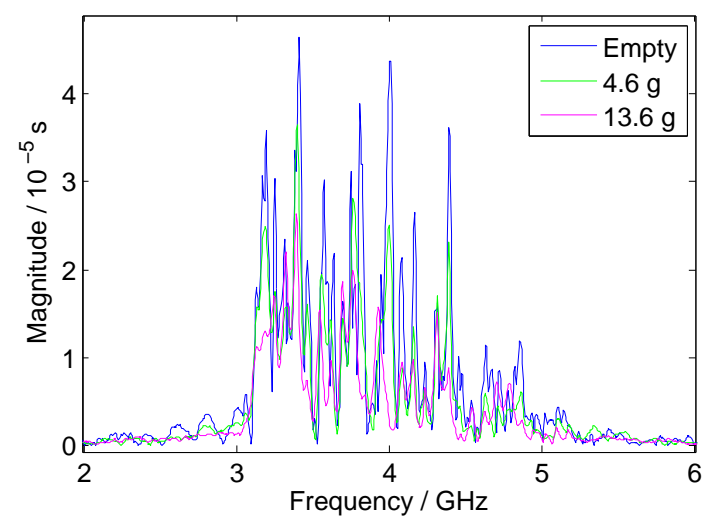

Figure 12. UWB magnitude spectrum for three different DPF loading states corresponding to the time-domain signals in Fig. 4.

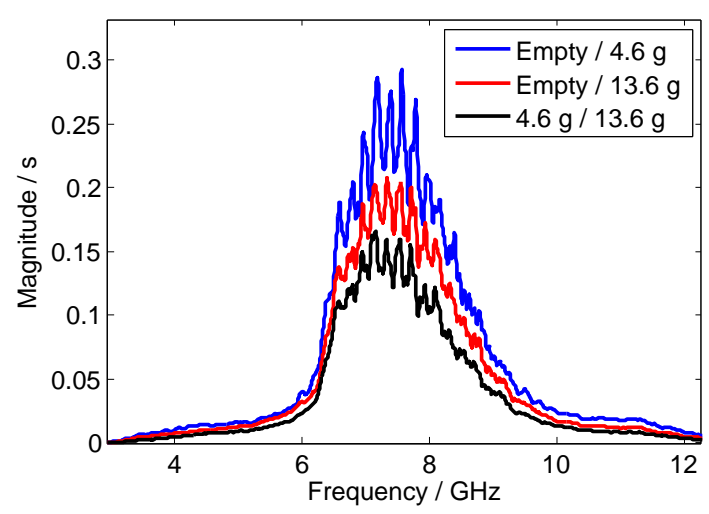

Figure 13. Convolution of the UWB magnitude spectra from Fig. 12.

Figure 14 shows the auto-correlation functions of the UWB magnitude spectra from Fig. 12, calculated with

$$
\begin{aligned}
& \Phi_{i j}(f)=\int_{-\infty}^{+\infty} U_{i}(\varphi) U_{j}(f+\varphi) \mathrm{d} \varphi \text { or } \\
& \Phi_{i j}(k \Delta f) \approx \sum_{\ell=\ell_{\min }}^{\ell_{\max }} U_{i}(\ell \Delta f) U_{j}((k+\ell) \Delta f) \Delta f,
\end{aligned}
$$

and $i=j$. With increasing soot load, the magnitude of these auto-correlation functions decreases. The maximum magnitude observed was $0.49,0.28$, and $0.16 \mathrm{~s}$ for the empty DPF, the DPF loaded with $4.6 \mathrm{~g}$ soot, and the DPF loaded with $13.6 \mathrm{~g}$, respectively. As in the time-domain case, the difference is also observable via the signal width.

Figure 15 presents the cross-correlation functions of the UWB magnitude spectra, Eq. (5) with $i \neq j$. The maximum magnitudes were $0.35,0.24$, and $0.19 \mathrm{~s}$ for the crosscorrelations of the three signals obtained with different soot loads.

Furthermore, we have prepared histograms of the spectral magnitudes, measured in A-D counts, for three different 


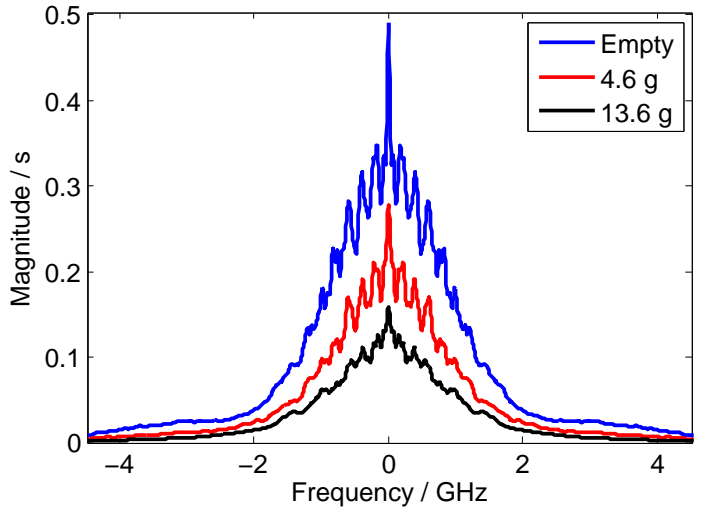

Figure 14. Auto-correlation functions of the UWB magnitude spectra in Fig. 12.

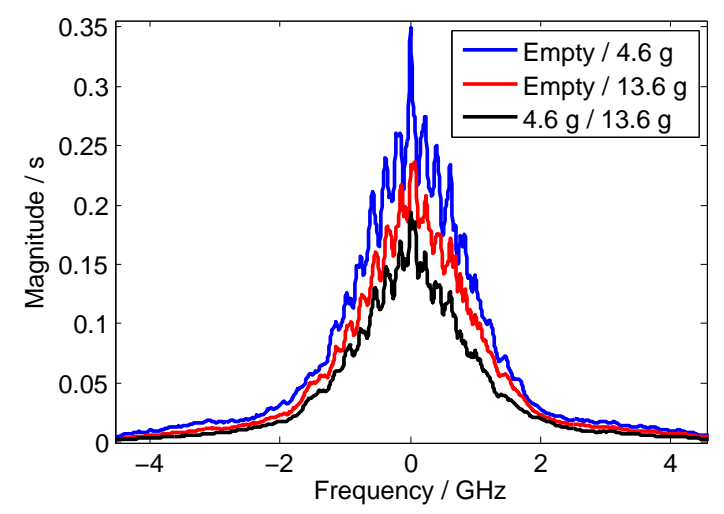

Figure 15. Cross-correlation functions of the UWB magnitude spectra in Fig. 12.

DPF loading states. The results are presented in Fig. 16. The frequency range evaluated was $3.1-5.3 \mathrm{GHz}$, consistent with the product specifications of the communication units used. The area of every rectangle in the histogram represents the relative frequency of the spectral magnitudes falling into the interval marked by the rectangle basis. The height of the rectangle is the relative frequency density (the relative frequency of the spectral magnitudes associated with the rectangle interval, divided by the width of the rectangle).

An appropriate empirical probability density function (pdf) used to fit the actually observed histogram must be one-sided and supported on the interval $[0, \infty]$ because the spectral magnitudes are non-negative numbers. Possible candidates, among others, are the Pareto distribution and the exponential distribution, both of which are often used in connection with system failure models. Here, we used the exponential distribution

$f(x ; \beta)=\left\{\begin{array}{cc}\frac{1}{\beta} e^{-x / \beta} & \text { for } x>0 \\ 0 & \text { for } x<0\end{array}\right.$.

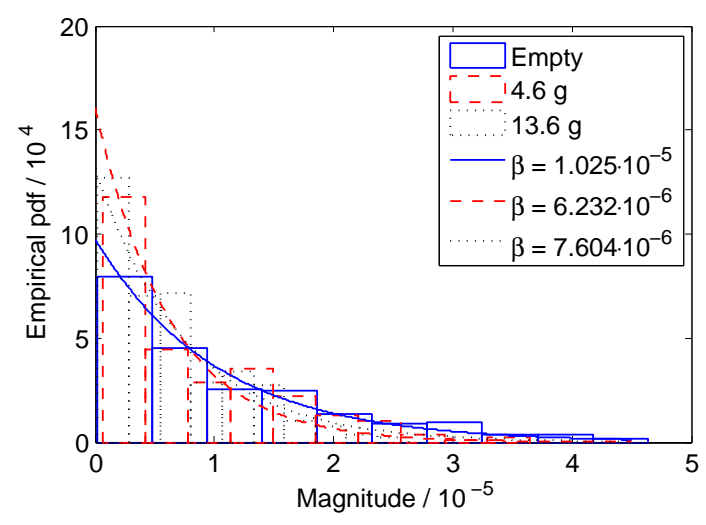

Figure 16. Histogram of the UWB magnitude spectrum for different loading states (bars) and best fits by exponential distributions $p(x)=\beta^{-1} e^{-x / \beta}$ (lines).

The exponential distributions with best-fitting parameters $\beta$ are also shown in Fig. 16. The widths of the histograms decrease with the soot content or, in other words, the standard deviation of the pdf decreases with the soot content. As before, this is to be expected: more soot means higher attenuation, which in turn results in smaller signal amplitudes at the receiver.

One would expect that more soot load (i.e., higher attenuation) would smooth the spectrum in the sense that the magnitude variations become smaller; this would correspond to a pdf graph with steeper skirts and smaller width. In numbers, this would mean that the pdf parameter $\beta$ increases with the soot load. The graphs in Fig. 16 support this assumption in principle (compare the 0 and $13.6 \mathrm{~g}$ soot cases), but one cannot infer a clear quantitative trend (compare the 4.6 and $13.6 \mathrm{~g}$ soot cases). On the other hand, given the small number of cases studied so far (only three different soot load values), there is not enough evidence to rule out the existence of a useful relationship between the soot load and the properties of the pdf describing the signal spectrum picked up by the receiver.

Finally, in analogy with the approach visualized for the time domain in Fig. 11, Fig. 17 depicts the maximum and average values for the magnitude spectrum in the range between 3.1 and $5.1 \mathrm{GHz}$. As before, the curves look promising in that they are monotonic and would allow for a unique inversion of the form "signal feature $\rightarrow$ soot load".

\section{Conclusions}

We have reported on a novel approach for the monitoring of exhaust gas aftertreatment systems, such as DPFs, SCRs, and TWCs, the most attractive feature of which is that it is based on a cost-effective modular architecture. Hence, it can be implemented in situ, e.g., in a car.

In the approach proposed, the interior of the catalyst metal housing serves as a communication channel between two 


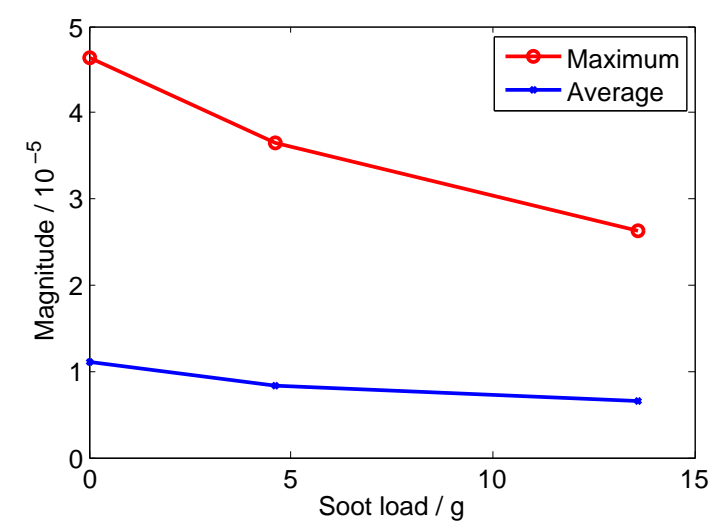

Figure 17. Maximum and average magnitudes of the signal spectrum at the receiver as functions of the soot load (from Fig. 12).

wireless communication nodes. To be more specific, we investigated a UWB-based architecture. It was demonstrated experimentally by way of various soot-loaded DPFs that the catalyst chemistry (i. e., the soot load of the DPF) affects the channel characteristics strongly enough to allow for the identification of the DPF state from the UWB signal details in the time as well as the frequency domains.

In particular, we were able to show that, in principle, certain features of the received time- and frequency-domain signals depend in a manner on the soot load which allows for the inversion of the feature vs. soot load characteristics. Among these features were the position of the center of gravity of the area bounded by the signal envelope and the time axis, the maximal value, the average value of the envelopes in the time domain and the maximum and average values in the spectra.

Acknowledgements. The authors thankfully acknowledge financial support by the German Research Foundation (DFG) under grants Fi 956/3-2 and Fi 956/5-1.

Edited by: F. L. Dickert

Reviewed by: two anonymous referees

\section{References}

Eichelbaum, M., Stößer, R., Karpov, A., Dobner, C.-K., Rosowski, F., Trunschke, A., and Schlögl, R.: The microwave cavity perturbation technique for contact-free and in situ electrical conductivity measurements in catalysis and materials science, Phys. Chem. Chem. Phys., 14, 1302-1312, doi:10.1039/c1cp23462e, 2012.

Feulner, M., Hagen, G., Piontkowski, A., Müller, A., Fischerauer, G., Brüggemann, D., and Moos R.: In-Operation Monitoring of the Soot Load of Diesel Particulate Filters: Initial Tests, Topics in Catalysis, 56, 483-488, doi:10.1007/s11244-013-0002-9, 2013.

Fischerauer, G., Spörl, M., Gollwitzer, A., Wedemann, M., and Moos, R.: Catalyst state observation via the perturbation of a microwave cavity resonator, Frequenz, 62, 180-184, doi:10.1515/freq.2008.62.7-8.180, 2008.
Fischerauer, G., Förster, M., and Moos, R.: Sensing the soot load in automotive diesel particulate filters by microwave methods, Meas. Sci. Technol., 21, 035108, doi:10.1088/09570233/21/3/035108, 2010a.

Fischerauer, G., Spörl, M., Reiß, S., and Moos, R.: MicrowaveBased Investigation of Electrochemical Processes in Catalysts and Related Systems, Technisches Messen, 77, 419-427, doi:10.1524/teme.2010.0066, 2010b (in German).

Marko, H.: Methoden der Systemtheorie, 2nd Edn., Springer, Berlin, Germany, 1982 (in German).

Moos, R., Beulertz, G., Reiß, S., Hagen, G., Fischerauer, G., Votsmeier, M., and Gieshoff, J.: Overview: Status of the Microwave-Based Automotive Catalyst State Diagnosis, Top. Catal., 56, 358-364, doi:10.1007/s11244-013-9980-x, 2013.

Motroniuk, I., Królak, R., Stöber, R., and Fischerauer, G.: State Observation in Automotive Aftertreatment Systems Based on Wireless Communication Links, in: Proc. 5th IMEKO TC19 Symp. on Environmental Instrumentation and Measurements, Chemnitz, Germany, 23-24 September 2014, 122-126, 2014.

Motroniuk, I., Stöber, R., and Fischerauer, G.: Waveform-Based State Determination for Catalytic Converters, in: Proc. 17th Int'1 Conf. on Sensors and Measurement Technology (Sensor 2015), Nuremberg, Germany, 19-21 May 2015, 472-477, 2015.

Ochs, T., Schittenhelm, H., Genssle, A., and Kamp, B.: Particulate Matter Sensor for On Board Diagnostics (OBD) of Diesel Particulate Filters (DPF), SAE Int. J. Fuels Lubr., 3, 61-69, doi:10.4271/2010-01-0307, 2010.

Reiß, S., Wedemann, M., Moos, R., and Rösch, M.: Electrical In Situ Characterization of Three-Way Catalyst Coatings, in: Proc. 8th Int'l Congress on Catalysis and Automotive Pollution Control (CAPOC 8), Brussels, Belgium, 15-17 April 2009, 3, 67-74, 2009.

Rose, D. and Boger, T.: Different Approaches to Soot Estimation as Key Requirement for DPF Applications, SAE Technical Paper, 2009-01-1262, 11 pp., doi:10.4271/2009-01-1262, 2009.

Sappok, A., Bromberg, L., Parks, J., and Prikhodko, V.: Loading and Regeneration Analysis of a Diesel Particulate Filter with a Radio Frequency-Based Sensor, SAE Technical Paper, 2010-01-2126, 14 pp., doi:10.4271/2010-01-2126, 2010.

Schödel, S., Moos, R., Votsmeier, M., and Fischerauer, G.: SIEngine Control With Microwave-Assisted Direct Observation of Oxygen Storage Level in Three-Way Catalysts, IEEE Trans. CST, 22, 2346-2353, 2014a.

Schödel, S., Votsmeier, M., and Fischerauer, G.: Microwaveassisted oxygen storage level estimation for three-way catalyst control: Model-based development and benchmarking of selected control strategies, Can. J. Chem. Eng., 92, 1597-1606, 2014b.

Twigg, M. V.: Progress and future challenges in controlling automotive exhaust gas emissions, Appl. Catal. B: Environmental, 70, 2-15, doi:10.1016/j.apcatb.2006.02.029, 2007.

Zimmermann, C.: Neuartiger Sensor zur Bestimmung des Zustandes eines NOx-Speicherkatalysators, PhD Thesis, Univ. Bayreuth, Shaker, Aachen, Germany, 2007 (in German). 